\section{Symmetry and the Monster}

by Mark Ronan

NEW YORK, OXFORD UNIVERSITY PRESS, 2006 255 PP. US\$27.00 ISBN 978-0-19-280722-9.

REVIEWED BY KISHORE MARATHE

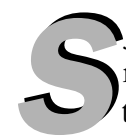

ymmetry and the Monster recounts the story of an exceptional result in the history of mathematics: The classification of finite simple groups. The existence and uniqueness of the largest sporadic group, dubbed the Monster, was the last piece in the classification. The complete classification is arguably the greatest achievement of 20th century mathematics. In fact, it is unique in the history of mathematics: The result of hundreds of mathematicians working in many countries around the world for over a quarter century. This global initiative was launched by Daniel Gorenstein, whose book [5] is still an excellent general reference for this material.

We now describe the highlights of this fascinating story. The first four chapters introduce groups and their application in Galois's work. Recall that a group is called simple if it has no proper nontrivial normal subgroups. Thus, an Abelian group is simple if and only if it is isomorphic to one of the groups $\mathbf{Z}_{p}$, for $p$ a prime number. This is the simplest example of an infinite family of finite simple groups. Another infinite family of finite simple groups is the family of alternating groups $A_{n}, n>4$ that we study in the first course in algebra. These two families were known in the 19th century. The last of the families of finite groups, called groups of Lie type, were defined by Chevalley in the mid 20th century. Chapters 5 to 9 discuss this material. By the early 20th century, the Killing-Cartan classification of simple Lie groups defined over the field $\mathbf{C}$ of complex numbers had produced four infinite families and five exceptional groups. This classification starts by classifying simple Lie algebras over $\mathbf{C}$ and then constructing corresponding simple Lie groups. In 1955, using this structure but replacing the complex numbers by a finite field, Chevalley's fundamental paper showed how to construct finite groups of Lie type. This work led to the classification of all infinite families of finite simple groups.

However, it was known that there were finite simple groups, called sporadic groups, that did not belong to any of these families. Chapters 10 to 14 are devoted to the discoveries of the 26 sporadic groups. The first sporadic group was constructed by Mathieu in 1861. In fact, he constructed five sporadic groups, now called Mathieu groups. There was an interval of more than 100 years before the sixth sporadic group was discovered by Janko in 1965. Two theoretical developments played a crucial role in the search for new simple groups. The first of these appeared in Brauer's address at the 1954 ICM in Amsterdam. It gave the definitive indication of the surprising fact that general classification theorems would have to include sporadic groups as exceptional cases. In fact, Fischer discovered and constructed his first three sporadic groups in the process of proving such a classification theorem. Brauer's work made essential use of elements of order 2. The second came in 1961, when Feit and Thompson proved that every nonAbelian simple finite group contains an element of order 2. The proof of this one line result occupies an entire 255page issue of the Pacific Journal of Mathematics (Volume 13, 1963). Before the Feit-Thompson theorem, the classification of finite simple groups seemed to be a rather distant goal. This theorem and Janko's new sporadic group greatly stimulated the mathematics community to look for new sporadic groups.

John Leech had discovered his 24dimensional lattice while studying the problem of sphere packing. The Leech lattice provides the tightest sphere packing in 24 dimensions. (However, the sphere packing problem in other dimensions is still wide open.) Symmetries of the Leech lattice contained Mathieu's largest sporadic group. It also had a large number of symmetries of order 2. Leech believed that the symmetries of his lattice contained other sporadic groups as well. Leech was not a group-theorist and he could not get group-theorists interested in his lattice. But he did find a young mathematician (who was not a grouptheorist) to study his work. In 1968, John Conway was a junior faculty member at Cambridge. He quickly became a believer in Leech's ideas. He tried to get Thompson, the great guru of group-theorists, interested in his work. Thompson told him to find the size of the group of symmetries and then call him. Conway later remarked that he did not know that he was using a folk theorem which says: The two main steps in finding a new sporadic group are (i) find the size of the group of symmetries, and (ii) call Thompson. Conway worked very hard on this problem and soon came up with a number. This work turned out to be his big break. It changed the course of his life and has made him into a world-class mathematician. He called Thompson with his number. Thompson called back in 20 minutes and told him that half his number could be a possible size of a new sporadic group and that there were two other new sporadic groups associated with it. These three groups are now denoted by Co1, Co2, Co3 in Conway's honor. Further study by Conway and Thompson showed that the symmetries of the Leech lattice give 12 sporadic groups in all, including all five Mathieu groups. In the early 1970s, Conway started the ATLAS project to collect all essential information (mainly the character tables) about the sporadic groups and some others. The work continued into the early 1980s when all the sporadic groups were finally known.

After Conway's work, the next major advance in finding new sporadic groups came through the work of Berndt Fischer. Working under Baer, Fischer became interested in groups generated by transpositions. Recall that, in a permutation group, a transposition interchanges two elements. Fischer first proved that a group $G$ generated by such transpositions falls into one of six types. The first type is a permutation group and the next four lead to known families of simple groups. It was the sixth case that led to three new sporadic groups, each related to one of the three largest Mathieu groups. The geometry underlying the 
construction of $G$ is that of a graph associated to generators of $G$. Permutation groups and the classical groups all have natural representations as automorphism groups of such graphs. Fischer's graphs give not only some known groups, but also his three new sporadic groups. He published this work in 1971 as the first of a series of papers; no further papers in the series ever appeared. In fact most of his work is not published. Fischer continued studying other transposition groups. This led him first to a new sporadic group, now called the Baby Monster. By 1981, 20 new sporadic groups were discovered, bringing the total to 25 . The existence of the 26th and the largest of these groups was conjectured independently by Fischer and Griess in 1973. Several scientists conjectured that this exceptional group must have relations with other areas of mathematics as well as with theoretical physics. The results that have poured in since then seem to justify this early assessment. Some strange coincidences noticed first by MacKay and Thompson were investigated by Conway and Norton. They called this group the Monster and their unbelievable set of conjectures "Monstrous Moonshine." Their paper [2] appeared in the Bulletin of the London Mathematical Society in 1979. The same issue of the Bulletin contained three papers by Thompson discussing his observations of some numerology between the Fischer-Griess Monster M and the elliptic modular functions. Thompson stated his conjectures about the relation of the characters of the Monster and Hauptmoduls for various modular groups. $\mathrm{He}$ also showed that there is at most one group which possesses the properties expected of $M$ and has a complex, irreducible representation of degree $196883=47.59 .71(47,59$ and 71 are the three largest prime divisors of the order of the Monster group). Conway and Norton had conjectured earlier that the Monster should have a complex, irreducible representation of degree 196883. Based on this conjecture, Fischer, Livingstone and Thorne (Birmingham notes 1978) computed the entire character table of the Monster.

The construction of the Monster was announced by Griess in 1981, and the complete details were given in [6]. Griess first constructed a commutative, nonassociative algebra $A$ of dimension 196884 and then showed that the Monster group is its automorphism group. In the same year, the final step in the classification of finite simple groups was completed by Norton by establishing that the Monster has an irreducible complex representation of degree 196883 (the proof appeared in print later). Combined with the earlier result of Thompson, this proved the uniqueness of the Monster. So the classification of finite simple groups was complete. The various parts of the classification proof together fill thousands of pages. The project to organize all this material and to prepare a flow chart of the proof is expected to continue for years to come.

The last three chapters give a brief account of the construction of the Monster and the Monstrous Moonshine Conjectures. We now give a mathematical formulation of these conjectures.

\section{Monstrous Moonshine Conjectures}

1. For each $g \in M$ there exists a MacKay-Thompson series $T_{g}(z)$ with normalized Fourier series expansion given by

$T_{g}(z)=q^{-1}+\sum_{1}^{\infty} c_{g}(n) q^{n}, q=e^{2 \pi i z}$.

There exists a sequence $H_{n}$ of representations of $M$, called the head representations, such that

$$
c_{g}(n)=\chi_{n}(g)
$$

where $\chi_{n}$ is the character of $H_{n}$.

2. For each $g \in M$, there exists a Hauptmodul $J_{g}$ for some modular group of genus zero, such that $T_{g}=J_{g}$. In particular,

(a) $T_{1}=J_{1}=J$, the Jacobi Hauptmodul for the modular group $\Gamma$.

(b) If $g$ is an element of prime order $p$, then $T_{g}$ is a Hauptmodul for the modular group $G_{p}$ studied by Ogg.

3. Let $[g]$ denote the set of all elements in $M$ that are conjugate to $g^{i}, i \in \mathbf{Z}$. Then $T_{g}$ depends only on the class $[g]$. Note that from Equation (1) and
(2), it follows that $T_{g}$ is a class function in the usual sense. However, $[g]$ is not the usual conjugacy class. There are 194 conjugacy classes of $M$ but only 171 distinct MacKay-Thompson series.

Conway and Norton calculated all the functions $T_{g}$ and compared their first few coefficients with the coefficients of known genus-zero Hauptmoduls. Such a check turns out to be part of Borcherds's proof, which he outlined in his lecture at the 1998 ICM in Berlin [1]. The first step was the construction of the Moonshine Modul. The entire book [3] by Frenkel, Lepowsky, and Meurman is devoted to the construction of this module, denoted by $V^{\natural}$. It has the structure of an algebra called the Moonshine vertex operator algebra (also denoted by $V^{\natural}$ ). They proved that the automorphism group of the infinite dimensional graded algebra $V^{\natural}$ is the largest of the finite, sporadic, simple groups, namely, the Monster.

The second step was the construction by Borcherds of the Monster Lie algebra using the Moonshine vertex operator algebra $V^{\natural}$. He used this algebra to obtain combinatorial recursion relations between the coefficients $c_{g}(n)$ of the MacKay-Thompson series. It was known that the Hauptmoduls satisfied these relations and that any function satisfying these relations is uniquely determined by a finite number of coefficients. In fact, checking the first five coefficients is sufficient for each of the 171 distinct series. Thus all the "Monstrous Moonshine" conjectures are now parts of what we can call the "Moonshine Theorem." Its relation to vertex operator algebras, which arise as chiral algebras in conformal field theory and string theory, has been established. In spite of the great success of these new mathematical ideas, many mysteries about the Monster are still unexplained. A recent update on the Moonshine may be found in the book by Gannon [4].

We conclude this summary with a comment, a modification of the remarks made by Ogg in [8] when the existence of the Monster group and its relation to modular functions were still conjectures (strongly supported by computational evidence). Its deep significance for theoretical physics is still emerging. So mathematicians and physicists, young 
and old, should rejoice at the emergence of a new subject, guaranteed to be rich and varied and deep, with many new questions to be asked and many of the conjectured results yet to be proved. It is indeed quite extraordinary that a new light should be shed on the theory of modular functions, one of the most beautiful and extensively studied areas of classical mathematics, by the largest and the most exotic sporadic group, the Monster. That its interaction goes beyond mathematics, into areas of theoretical physics, such as conformal field theory, chiral algebras and string theory, may be taken as strong evidence for a new area of research which this reviewer has called in [7] "Physical Mathematics."

Symmetry and the Monster is written in nontechnical language and yet conveys the excitement of a great mathematical discovery usually accessible only to professional mathematicians. The author knew many of the contributors, and this brings a nice personal touch to the narrative. His use of nonstandard terminology seems quite unnecessary, however. The term "atom of symmetry" is not more illuminating than "simple group" for the lay reader and is annoying to anyone who has taken a first course in algebra. There are several factual errors and misstatements. The worst puts Newton and Leibniz developing calculus in the 16th century (p. 87) and again in the 17 th century (p. 89). Janos Bolyai's appendix is at the end of his father's book on geometry and not in the book by Gauss (p. 195). Parts dealing with physics, especially the last chapter, contain misstatements. There is no evidence at this time that string theory combines quantum physics and general relativity (p. 72) or that it provides a model for elementary particles (p. 218). The level of material varies greatly. It is doubtful that a reader who needs to be reminded of the quadratic formula, golden ratio or $\pi$ and $e$ will take away much mathematics from this book. But in spite of these shortcomings, the book gives a good description of many aspects of an important event in the history of mathematics.

\section{OPEN ACCESS}

This article is distributed under the terms of the Creative Commons Attribution Noncommercial License which permits any noncommercial use, distribution, and reproduction in any medium, provided the original author(s) and source are credited.

\section{REFERENCES}

[1] R. E. Borcherds. Monstrous moonshine and monstrous Lie superalgebras. Inventiones Math., 109:405-444, 1992.

[2] J. H. Conway and S. P. Norton. Monstrous moonshine. Bull. London Math. Soc., 11(3):308-339, 1979.
[3] I. Frenkel, J. Lepowsky, and A. Meurman. Vertex Operator Algebras and the Monster. Pure and App. Math., \# 134. Academic Press, New York, 1988.

[4] T. Gannon, Moonshine Beyond the Monster. Cambridge University Press, Cambridge, 2006.

[5] D. Gorenstein, Finite Simple Groups. Plenum Press, New York, 1982.

[6] R. Griess. The friendly giant. Invent. Math., 69:1-102, 1982.

[7] Kishore Marathe. A Chapter in Physical Mathematics: Theory of Knots in the Sciences. In: B. Engquist and W. Schmidt eds., Mathematics Unlimited-2001 and Beyond, pp. 873-888, Berlin, 2001. Springer-Verlag.

[8] A. P. Ogg, Modular functions. In Santa Cruz Conference on Finite Groups, Proc. Sympos. Pure Math., 37, pp. 521532, Providence, 1980. Amer. Math. Soc.

Department of Mathematics

City University of New York

Brooklyn College

Brooklyn, NY 11210, USA

e-mail: kmarathe@brooklyn.cuny.edu;

marathe@mis.mpg.de

Max Planck Institute for Mathematics in the Sciences Leipzig

Dresden, Germany 